\title{
An open level study to assess the glycemic control effect of metformin and Pioglitazone as add on therapy along with sulfonylurea in uncomplicated type 2 diabetes mellitus
}

\author{
Goutameswar Mazumdar*, Bikash Swaika, Anindya Dasgupta \\ Burdwan Medical College, Burdwan, India; ${ }^{*}$ Corresponding Author: goutameswar09@gmail.com
}

Received 20 January 2012; revised 16 March 2012; accepted 10 April 2012

\section{ABSTRACT}

Introduction: Management of hyperglycemia in type2 diabetes mellitus (T2DM) becomes the top priority. When single antidiabetic drug is ineffective, combination is required for good glycemic control. There is a dearth of studies that provide head to head comparison of the ability of combinations and therefore need further study. Objectives: To assess and compare the glycemic control and physical parameter altering effect of glibenclamide, glibenclamide \& Pioglitazone, glibenclamide \& metformin in T2DM. Methods and materials: 100 T2DM patients were selected from outpatients department of medicine following prefixed inclusion and exclusion criteria. Fasting and postprandial blood glucose (fbg \& ppbg) and physical parameters (waist, hip and thigh circumference) were measured before and after treatment with study drugs and adverse effects of these drugs were recorded. Data were analyzed by employing paired $t$-test and chi-square test. Results: 11 patients lost the follow up. A some total of $\mathbf{8 9}$ middle aged, predominantly male, non obese T2DM patients after exposure to the study drugs showed significant $(p<0.05)$ reduction of blood glucose from baseline. Reduction of blood glucose and waist:hip ratio were observed significantly $(p<0.05)$ more with glibenclamide and metformin combination with some tolerable side effects. Discussion: Metformin and Pioglitazone both are insulin sensitizer but metformin \& glibenclamide combination showed significantly $(p<0.001)$ more reduction of fbg, ppbg and central obesity (waist: hip ratio) than Pioglitazone \& glibenclamide combination. Therefore Judicious use of low dose of glibenclamide and full dose of metfor- min become safe, effective and cheap for the treatment of type 2 diabetes patients in poor country like India.

Keywords: Type 2 Diabetes; Glycemic Control; Combination Therapy; Waist: Hip Ratio

\section{INTRODUCTION}

Type 2 diabetes mellitus (T2DM) is a leading cause of morbidity and mortality accounting for $90 \%$ of total diabetes cases globally [1]; and is a chronic and progressive disease demanding intense management from diagnosis $[2,3]$. The recognition that achieving specific glycemic goal can substantially reduce morbidity, have made the effective treatment of hyperglycemia a top priority [4]. Life style change and the use of pharmaceutical therapies are considered pivotal to achieve good control of hyperglycemia [5]. The selection of best medication or combinations of medication for the management of hyperglycemia in patients with T2DM, while based in science, remains to a large degree in the realm of "art" [6]. Unfortunately there is a dearth of high quality studies that provide head to head comparisons of the ability of medications to achieve the currently recommended glycemic level [7]. T2DM is associated with defect in insulin action (insulin resistance) and secretion where Insulin resistance is a consistent finding present years before the onset of diabetes [8]. So the treatment of T2DM should comprise of secretagouges to stimulate the secretion of insulin and Insulin sensitizer to facilitate insulin action. Decline in $\beta$-cell function, characteristic of T2DM, has been postulated to detrimental effects of insulin resistance [9]. Metformin (MET) and Pioglitazone (PIO) are the commonly used insulin sensitizer. MET Suppresses hepatic gluconeogenesis, reduces the effect of glucagon, increases insulin mediated muscle glucose uptake and utilization, increases the translocation of insulin sensitive 
glucose transporters into the cell membrane and promotes the insulin and glucose sensitive transport properties of glucose transporters [10]. PIO activates the Peroxisome Proliferator-Activated Receptor Gama (PPAR- $\gamma$ ) receptor, regulates adipocyte differentiation, improves lipid homeostasis and insulin action, reduces insulin resistance, decreases the release of free fatty acids and cytokines, increases adiponectin, augments glucose transport in muscle, raises glycogen synthesis in liver, generates nitric oxide in vascular endothelium and slow the rate of loss of $\beta$-cell insulin secretary function [11]. Both have several beneficial effects on T2DM management. Therefore the purpose of this study is to make a head to head comparison of the ability of these two insulin sensitizer in reducing hyperglycemia and altering waist hip ratio when used individually along with sulfonylurea as add on therapy among uncontrolled T2DM patients. The objectives of this study were to assess the glycemic control effect and to compare the waist: hip ratio altering effect of glibenclamide (GBCM), glibenclamide \& metformin (GBCM \& MET) combination, glibenclamide \& Pioglitazone (GBCM \& PIO) combination when used sequentially in the same group of T2DM patients.

\section{MATERIALS AND METHODS}

This study was conducted in the out patients department of Medicine and dept of Biochemistry and Pharmacology of Burdwan Medical College \& Hospital for a period of 6months in the year 2009-2010. 100 T2DM patients of both sexes and all ages not controlled by monotherapy were selected randomly for this study. The exclusion criteria were unable to give informed consent, renal impairment, hepatic disease, heart failure, lactic acidosis, alcoholics, pregnancy, female subjects planning for pregnancy, subjects having chronic hypoxic lung disease, taking low calorie diet and $\mathrm{h} / \mathrm{o}$ long standing repeated fasting. This study was approved by the institutional ethics committee. Written informed consent was taken from all the study subjects. The study design was open level self controlled sequential interventional prospective study. Study was conducted by following the Helsinki declaration 1975 and ICMR guideline. Patients were selected randomly and all were advised to follow the medical nutrition therapy and life style modification as per direction; and oral antidiabetic drug was change to glibenclamide (GBCM) as sulfonylurea at a dose of $5 \mathrm{mg}$ or $10 \mathrm{mg}$ once daily according to the patient in a period of two weeks; and fasting and postprandial blood glucose (fbg \& ppbg) and physical parameters (waist, hip and thigh circumference) were measured as base line for all the study patients. Fbg \& ppbg and other physical parameters were measured after four weeks of glibenclamide therapy. Then, PIO was started initially at a dose of
$15 \mathrm{mg} /$ day along with previous same dose of GBCM and dose escalation of PIO was done up to $30 \mathrm{mg}$ /day within a period of two weeks. Then after six weeks fbg \& ppbg and other physical parameters were measured. PIO was then withdrawn from all patients keeping GBCM at the same previous dose for a period of two weeks (washout period of PIO). Then MET was added, along with the same previous dose of GBCM, initially $850 \mathrm{mg}$ once daily dose, later dose escalation was done gradually with the maximum dose of $850 \mathrm{mg}$ three times daily(total $2550 \mathrm{mg} /$ day) in a period of two weeks. Then after six weeks fbg \& ppbg and other physical parameters were measured. Adverse effects of all these drugs were monitored in all visits during the whole study period by using standard case reporting form of adverse drug reaction. Study subjects were trained regarding the signs and symptoms of hypoglycemia at the beginning of the study and were asked to inform immediately if they feel any such at any time. All the observed data was analyzed critically.

Statistical analysis: Data has been presented with mean and standard deviation. Paired $t$-test has been employed to test the significance of difference of glycemic control and physical parameter alteration. Chi-square test has been used to test the significance of association of adverse effects produced by GBCM + PIO and GBCM + MET combination. For all analyses $p$-value $<0.05$ has considered as statistically significant. Statistical analysis has performed by using spss -17 software.

\section{RESULTS}

Among the total 100 patients only 89 patients had completed this study and were accepted for analysis. There demographic profiles are mentioned in Table 1. Study subjects were predominantly male $(65.17 \%)$, middle aged (mean age $47.91 \pm 10.5 \mathrm{yrs}$ ), Hindu (71.8\%), non-obese (mean BMI $20.97 \pm 4.86 \mathrm{~kg} / \mathrm{M}^{2}$ ), poor (per capita monthly income is Rs $853.81 /-$ ) and literate (83.1\%). Mean duration of diabetes was 4.89 yrs. Mean base line Insulin, fbg and ppbg level of all these patients were $11.61 \pm 4.61 \mu \mathrm{u} / \mathrm{ml}, 186.37 \pm 36.98 \mathrm{mg} / \mathrm{dl}$ and $274.68 \pm 47.89 \mathrm{mg} / \mathrm{dl}$ respectively. Family history of diabetes was positive in only 24 patients $(27 \%)$. Occupation was mixed like house wife, service and others. Rural and urban distribution of patients was 48 vs. 41 patients (53.9\% and $46.1 \%)$.

After completion of baseline examination intervention had been done by employing the study drugs sequentially and following results were obtained as mentioned in Table 2.

Results showed that fbg \& ppbg was effectively and significantly $(p<0.001)$ reduced from the base line by GBCM (SEM 3.43, 95\% CI 47.53 to 61.18 for fbg and 
Table 1. Demographic profile of the study subjects.

\begin{tabular}{cc} 
Number of patients & 89 \\
Age in years (mean $\pm \mathrm{SD})$ & $47.91 \pm 1058$ \\
Sex & Male-58, female-31 \\
Religion & Hindu-55, muslim-34 \\
Body weight in Kg (mean $\pm \mathrm{SD})$ & $53.95 \pm 10.65$ \\
Percapita monthly income in Rs (mean) & 853.81 \\
Duration of diabetes in years (mean) & 4.8977 \\
Family history of diabetes & Positive-24 cases and negative- 65 casses \\
Education & Literate-15 illeterate- 74 \\
Occupation & House wife-29, govt. service-20, others-40 \\
Residence & Urban- 41, rural-48 \\
Body Mass Index in $\mathrm{Kg} / \mathrm{m}^{2}($ mean $\pm \mathrm{SD})$ & $20.97 \pm 4.86$ \\
\hline
\end{tabular}

Note: SD: standard deviation, $\mathrm{kg} / \mathrm{m}^{2}$ : kilogram/square meter of body height.

Table 2. Change of values from baseline after therapy with GBCM, GBCM \& PIO, GBCM \& MET.

\begin{tabular}{|c|c|c|c|c|c|}
\hline character & $\begin{array}{c}\text { Base line } \\
\text { (mean with SD) }\end{array}$ & $\begin{array}{c}\text { GBCM } \\
\text { (mean with SD) }\end{array}$ & $\begin{array}{l}\text { GBCM\&PIO } \\
\text { (mean with SD) }\end{array}$ & $\begin{array}{l}\text { GBCM\&MET } \\
\text { (mean with SD) }\end{array}$ & $P$-value ${ }^{*}$ \\
\hline Fbg (mg/dl) & $186.37 \pm 36.98$ & $132.02 \pm 21.94$ & $119.08 \pm 19.52$ & $104.15 \pm 15.67$ & 0.000 \\
\hline Ppbg (mg/dl) & $274.68 \pm 47.89$ & $197.39 \pm 32.71$ & $177.23 \pm 28.59$ & $156.30 \pm 19.46$ & 0.000 \\
\hline Waist cir (in inch) & $31.97 \pm 3.90$ & $32.80 \pm 3.78$ & $32.56 \pm 3.85$ & $31.01 \pm 3.74$ & 0.000 \\
\hline Hip cir (in inch) & $34.00 \pm 4.05$ & $34.47 \pm 4.03$ & $34.08 \pm 4.11$ & $33.37 \pm 3.98$ & 0.000 \\
\hline $\mathrm{w} / \mathrm{h}$ ratio & 0.944 & 0.955 & 0.961 & 0.933 & 0.000 \\
\hline Thigh cir (inch) & $16.44 \pm 1.64$ & $16.46 \pm 1.65$ & $16.47 \pm 1.64$ & $16.41 \pm 1.63$ & 0.068 \\
\hline
\end{tabular}

Note: fbg-fasting blood glucose, ppbg-postprandial blood glucose, w/h ratio: waist:hip ratio, SD: standard deviation, mg/dl: milligram/deciliter, cir: circumference, ${ }^{*} p$-value of paired $t$-test between GBCM \& PIO and GBCM \& PIO group.

SEM 4.44, 95\% CI 68.45 to 86.13 for ppbg), by GBCM \& PIO (mean reduction $67.29 \mathrm{mg} \%$, SEM $3.43,95 \% \mathrm{CI}$ 60.45 - 74.12, for fbg and mean reduction $97.44 \mathrm{mg} \%$; SEM 4.46; and 95\% CI 88.56 - 106.33 for ppbg), by GBCM \& MET (mean reduction 82.22 mg\%, SEM 3.34, 95\% CI $75.58-88.86$ for fbg and mean reduction 118.38 mg\% SEM 4.18; and 95\% CI 110.7 - 126.69 for ppbg) when tested with paired $t$-test. Such reduction was observed significantly more with GBCM \& MET than GBCM \& PIO and GBCM therapy. Waist circumference was reduced significantly $(p<0.001)$ by the GBCM \& MET from $31.97 \pm 3.90$ inch (base line) to $31.01 \pm 3.74$ inch where as it was modestly increased by GBCM $(32.80 \pm 3.78$ inch) and GBCM \& PIO (32.56 \pm 3.85 inch). Similar results were also observed with thigh circumference but not statistically significant. Waist hip ratio was significantly reduced from base line by GBCM $\& \operatorname{MET}(p<0.001$, mean reduction $0.01105 \pm 0.03105$, SEM 0.00329 , 95\% CI 0.00450 - 0.01759) but it was raised by GBCM alone and GBCM \& PIO.

Some adverse effects were found following drug therapy among the study subjects and they are as mentioned in Table 3.

Flatulence, dyspepsia, distaste, loss of appetite were significantly more with GBCM \& MET combination than GBCM \& PIO combination. But all these sign and symptoms disappeared shortly during study and none discontinued the therapy.

\section{DISCUSSION}

Non-obese middle aged male was predominant in this study though it is common in women [12]. Male preponderance, increased drop out rate $(11 \%)$ and two weeks interval visit in this study are the reflection of outpatient department based hospital study. Sulfonylurea is arguably the most cost effective glucose lowering agents [12]. GBCM, a low cost sulfonylurea, one of the 
Table 3. The adverse effects observed in this study following GBCM, GBCM + MET, GBCM + PIO therapy.

\begin{tabular}{|c|c|c|c|c|}
\hline Adverse effects & $\operatorname{GBCM}(\%)$ & GBCM + MET (\%) & $\mathrm{GBCM}+\mathrm{PIO}(\%)$ & $\mathrm{X}^{2}$-test \\
\hline Hypoglycemia & Nil & Nil & Nil & - \\
\hline Weight gain & Mild & Reduced Bwt & Mild & - \\
\hline Flatulence (n) & Nil & $18(20.22 \%)$ & $3(3 \%)$ & 0.001 \\
\hline Dyspepsia (n) & Nil & $26(39.21 \%)$ & $3(3 \%)$ & 0.000 \\
\hline Distaste (n) & $7(0.08 \%)$ & $23(25.84 \%)$ & $4(4 \%)$ & 0.000 \\
\hline Loss of appetite (n) & Nil & $19(21.35 \%)$ & $7(8 \%)$ & 0.018 \\
\hline Diarrhea (n) & Nil & $11(12.35 \%)$ & $5(5 \%)$ & 0.189 \\
\hline Anemia (n) & Nil & Nil & Nil & - \\
\hline Edema (n) & Nil & Nil & $3(3 \%)$ & - \\
\hline Sgpt level & Normal & Normal & Mildly raised in 8 cases $(9 \%)$ & - \\
\hline
\end{tabular}

Note: n: number of patients, GBCM: glibenclamide, MET: metformin, PIO: Pioglitazone, $\mathrm{x}^{2}$ test: chi-square Exat sig (2-sided) test.

commonly prescribed insulin secretagouges [13], approved for monotherapy, begin to lower plasma glucose immediately, cause similar degree of glycemic control $(1 \%-2 \% \mathrm{HbA} 1 \mathrm{c})$ and is likely beneficial [12]. Again at higher doses GBCM modestly reduces the blood glucose, increases the incidence of hypoglycemia and might cause increase arrhythmic cardiovascular events so need to avoid high-dose therapy [14]. Considering these facts GBCM was used at low dose (5 or $10 \mathrm{mg} / \mathrm{d})$ in this study. MET and PIO are able to reduce the fbg by $58 \mathrm{mg} / \mathrm{dl}$ and $65 \mathrm{mg} / \mathrm{dl}$ and $\mathrm{HbAlc}$ by $1.8 \%$ and $1.6 \%$ respectively at maximum doses [15]. Base line fbg was187.21 \pm 38.1 $\mathrm{mg} \%$ and $\mathrm{HbA} 1 \mathrm{c}$ was $8.62 \%$. Therefore as monotherapy MET and PIO individually assumed to be insufficient to reduce blood glucose near normal in this study. GBCM significantly decreased the blood glucose here but not to the near normal. Again the goal of all patients should be to lower blood glucose levels to as close to the normal range as safety and life style allow [16] and therefore intensive therapy has been endorsed as treatment of choice for most of the diabetics [17]. $\beta$-cell dysfunction is also reversible to a large degree by intensive glycemic control [16]. So it is wise to use the combination of sulfonylurea and insulin sensitizer to keep the blood glucose normal and to reduce the dose of sulfonylurea for safety. GBCM \& MET and GBCM \& PIO combination therapy in the same cohort of patients had significantly $(p<$ 0.001 ) reduced the blood glucose. But GBCM \& MET brought it near normal where as GBCM \& PIO maintained it above the normal level. According to the projections from UKPDS, $1 \%$ decrement in $\mathrm{HbAlc}$ is associated with a $14 \%$ reduction in risk of heart attacks and a $37 \%$ reduction in risk of micro vascular complications
[18]. The defect in T2DM primarily is defective regulation of glucose production from the liver by glycogenolysis and gluconeogenesis. It was found that hepatic gluconeogenesis accounted for $50 \%$ to $96 \%$ of glucose production and the percentage increased with increasing duration of fasting [19]. Metformin reduces hepatic insulin resistance thereby gluconeogenesis through AMP-activated protein kinase [20]. Again GBCM \& MET had reduced waist circumference (central obesity) and waist hip ratio significantly $(p<0.001)$ and secondarily further reduced the insulin resistance. PIO through Peroxisome Proliferator-Activated Receptor Gama (PPAR- $\gamma$ ) activity improves insulin sensitivity thereby improves glycemic control and HDL, reduces plasma FFA and triglyceride by $20 \%$, modify LDL particle size and number [12]. However, in this study GBCM and GBCM \& PIO had increased the waist circumference and waist:hip ratio and may raise the possibility of increased insulin resistance. PPAR- $\gamma$ expressed more in adipose but muscle tissue utilize more the postprandial plasma glucose. Probably this is the reason of better glycemic control by the GBCM \& MET combination than GBCM and GBCM \& PIO. The occurrence of flatulence, dyspepsia, distaste, diarrhea, loss of appetite is more with the MET combination but these were minor incidence which improve in due course of time. Sgpt level increased with PIO combination though mild and less in number of case $(9 \%)$ still a matter of concern. Therefore it can be concluded that the judicious use of low dose GBCM and full dose metformin is a cost effective, safe and useful combination for poor Indian population in type 2 diabetes management as this combination reduced both fbg, ppbg, central obesity, waist:hip more than GBCM alone and 
GBCM \& PIO combination. Further MET is recommended as a core therapy in the diabetes management worldwide at diagnosis either alone or in combination with other oral anti diabetic therapies or insulin [9].

\section{REFERENCES}

[1] Zimmet, P., Alberti, K.G. and Shaw, J. (2001) Global and social implication of the diabetes epidemic. Nature, 414, 782-787. doi:10.1038/414782a

[2] American Diabetes Association. (2008) Standards of medical care in diabetes. Diabetes Care, 31, S12-S54. doi: $10.2337 / \mathrm{dc} 08-\mathrm{S} 012$

[3] Task Force Members. (2007) Guidelines on diabetes, prediabetes and cardiovascular diseases: Executive summary. The task force on diabetes and cardiovascular diseases of the European Society of Cardiology (ESC) and of the European Association for the Study of Diabetes (EASD) European Heart Journal, 28, 88-136

[4] UK Prospective Diabetes Study (UKPDS) Group. (1998) Intensive blood glucose control with sulfonylurea or insulin compared with conventional treatment and risk of complication in patients with type 2 diabetes (UKPDS 33). The Lancet, 352, 837-853. doi:10.1016/S0140-6736(98)07019-6

[5] Gaede, P., et al. (2008) Effect of a multifactorial intervention on mortality of type 2 diabetes. The New England Journal of Medicine, 358, 580-591. doi:10.1056/NEJMoa0706245

[6] White, J.R. and Campbell, R.K. (2009) Overview of medications used to treat type 2 diabetes. In: White, J.R. and Campbell, R.K., Eds., ADA/PDR Medication for the Treatment of Diabetes, The Healthcare Business of Thomson Reuters, Montvale, 5-15.

[7] Nathan, D.M., Buse, J.B., Davidson, M.B., et al. (2006) Management of hyperglycemia in type 2 diabetes mellitus: A consensus algorithm for the initiation and adjustment of therapy. In: White, J.R. and Campbell, R.K., Eds., ADA/PDR Medication for the Treatment of Diabetes, The Healthcare Business of Thomson Reuters, Montvale, 17-31.

[8] Warram, J.H., Martin, B.C., et al. (1990) Slow glucose removal rate and hyperinsulinemia precede the development of type 2 diabetes in the offspring of diabetic parents. Annals of International Medicine, 113, 909-915.

[9] Lebovitz, H.E. (2002) Rational for and role of thiazolidinediones in type 2 diabetes mellitus. The American Journal of cardiology, 90, 34-41. doi:10.1016/S0002-9149(02)02557-2

[10] Bailey, C.J. (2009) Metformin. In: Lebovitz, H.E., Ed.,
Therapy for Diabetes Mellitus and Related Disorders, 5th Edition, American Diabetes Association, Alexandria, 235-253.

[11] Lebovitz, H.E. (2009) Thiazolidinedione. In: Lebovitz, H.E., Ed., Therapy for Diabetes Mellitus and Related Disorders, 5th Edition, American Diabetes Association, Alexandria, 260-272.

[12] Buse, J.B., Polonsky, K.S. and Burant, C.F. (2008) Type 2 diabetes mellitus. In: Kronenberg, H.M., Melmed, S., Polanski, K.S., Larsen, P.R., Eds., William's Textbook of Endocrinology, 11th Edition, Saunders, Philadelphia, 1330-1389.

[13] Klezig, H., kober, G., Matter, C., et al. (1999) Sulfonylurea and ischemic preconditioning; a double blind placebo controlled evaluation of glimepiride and glibenclamide. European Heart Journal, 20, 439-446. doi:10.1053/euhj.1998.1242

[14] Riddle, M.C. (2003) Editorial sulfonylureas differ in effects on ischemic preconditioning-Is it time to retire glyburide? The Journal of Clinical Endocrinology Metabolism, 88, 528-530. doi:10.1210/jc.2002-021971

[15] Lebovitz, H.E. (2010) Management of hyperglycemia with oral antihyperglycemic agents in type 2 diabetes. In: Khan, C.R., Weir, G.C., King, G.L., Jacobson, A.M., Moses, A.C., Smith, R.J. Eds., Joslin's Diabetes Mellitus, 14th Edition, Wolters Kluwer Pvt Ltd., New Delhi, 685-710.

[16] UK Prospective Diabetes Study (UKPDS) Group (2005) Association of glycemia with micro vascular and macro vascular complications of type 2 diabetes (UKPDS 35): Prospective observational study. British Medical Journal, 32, 405-412.

[17] Vague, P. and Moulin, J.P. (1982) The defective glucose sensitivity of the $\beta$-cell in noninsulin dependent diabetes: Improvement after twenty hours normoglycemia. $\mathrm{Me}$ tabolism, 31, 139-142. doi:10.1016/0026-0495(82)90125-1

[18] Nathan, D.M. (2010) Relationship between metabolic control and long-term complications of diabetes. In: Khan, C.R., Weir, G.C., King, G.L., Jacobson, A.M., Moses, A.C., Smith, R.J. Eds., Joslin's Diabetes Mellitus, 14th Edition, Wolters Kluwer Pvt Ltd., New Delhi, 809-821.

[19] Rothman, D.L., Magnusson, I., Katz, L.D., et al. (1991) Quantitation of hepatic glycogenolysis and gluconeogenesis in fasting human with 13C NMR. Science, 254, 573-576.

[20] Long, Y.C. and Zierath, J.R. (2006) AMP-activated protein kinase signaling in metabolic regulation. The Journal of Clinical Investigation, 16, 1776-1783. doi:10.1172/JCI29044 